\section{Sclerosing encapsulating peritonitis}

\author{
Jen-Jung Pan, Nirav Thosani, \\ Michael B. Fallon
}

Division of Gastroenterology, Hepatology and Nutrition, Department of Internal Medicine, University of Texas Health Science Center at Houston, Houston, Texas, USA

\section{Abstract}

Sclerosing encapsulating peritonitis (SEP) is a rare cause of intestinal obstruction. This entity has been reported as either primary idiopathic or secondary to other diseases. We report SEP in 2 cirrhotic patients and review the literature. Both patients had decompensated cirrhosis and episodes of spontaneous bacterial peritonitis. One patient underwent a Denver shunt placement before developing SEP. This patient remains alive and is managed conservatively. The other patient deceased from multi-organ failure after the resection of gangrened small bowel. The manifestations of SEP are often nonspecific that leads to misdiagnosis and/or delayed diagnosis. Early diagnosis of SEP is difficult but not impossible. Surgical treatment is often required when intestinal obstruction is present. Nevertheless, patients with this problem can be treated conservatively with immunosuppressive therapy with or without total parenteral nutrition (TPN) before going for surgery.

\section{Introduction}

Sclerosing encapsulating peritonitis (SEP), or abdominal cocoon, is a rare cause of small bowel obstruction. In this condition, the bowel is partially or completely encased by a thick fibrous membrane forming several compartments with loops of small bowel inside. SEP can be classified either as idiopathic or secondary to conditions such as peritoneal dialysis (PD), lupus, sarcoidosis, familiar Mediterranean fever, and use of beta blockers. ${ }^{1}$ SEP has been reported in cirrhotic patients with ${ }^{2-4}$ or without peritoneal-venous shunt (PVS)..$^{5-7}$ It can also develop after liver transplantation (LT). ${ }^{8}$ We report here a case of SEP in 2 cirrhotic patients, including one fatality, and review the literature.

\section{Case Report \#1}

Patient 1 was a 51 -year-old female who has human immunodeficiency virus (HIV) infection on highly active antiretroviral therapy (HAART) and portal hypertension secondary to portal and splenic vein thrombosis that was diagnosed in April 2008. Because of her refractory ascites, she received a Denver shunt in February 2009. During the laparotomy, her liver appeared cirrhotic. However, the peritoneum appeared unremarkable. The shunt worked for 2 weeks only and then became occluded. She then went back to have frequent therapeutic paracentesis. She had bouts of spontaneous bacterial peritonitis (SBP). At 2 months prior to the hospitalization in September 2010, she developed worsening abdominal pain that was exacerbated after meals. She also developed intermittent nausea and vomiting. Her bowel movements alternated between constipation and diarrhea. On physical examination, there was an induration in her mid abdomen that was tender to palpation. A contrast-enhanced abdominal and pelvic computed tomography (CT) scan was performed during the hospitalization that showed interval development of well-circumscribed clustering of small bowel loops in the right mid-abdomen and pelvis that appeared to be surrounded by soft tissue density capsule. The nonencapsulated small bowel loops were slightly prominent without evidence of small bowel obstruction (Figure 1A). She also had a small bowel follow through study during the admission that revealed no passage of contrast into the colon at 4 hours representing delayed passage of contrast (Figure 1B). All the findings were consistent with SEP. In November 2010, she had the Denver shunt removed. She continues to receive conservative management for SEP with minimal symptoms currently.

\section{Case Report \#2}

Patient 2 was a 40 -year-old male who had a history of cirrhosis secondary to chronic hepatitis C. He underwent surgical repairs of the right inguinal and umbilical hernia in 2008 and presented with postprandial nausea and vomiting and abdominal pain to our hospital in early November 2010. A CT scan of abdomen and pelvis was performed that revealed a loculated fluid collection anteriorly surrounding the liver and extending to the left abdomen consistent with peritonitis. In addition, diffuse thickening of the small and large bowel was noted. Several dilated loops of proximal jejunum were seen in the mid left abdomen with surrounding fluid but without air-fluid level or evidence of high-grade obstruction. Surgical consultant suspected that he had a partial small bowel obstruction and did not need a surgical intervention. His diet was then advanced as tolerated. A diagnostic paracentesis was performed that confirmed the presence
Correspondence: Jen-Jung Pan, Division of Gastroenterology, Hepatology and Nutrition Department of Internal Medicine, University of Texas Health Science Center at Houston, 6431 Fannin Street, MSB 4.234, Houston, Texas 77030 USA.

Tel. +1.713.500.6677 - Fax: +1.713.500.6699.

E-mail: jenjung.pan@uth.tmc.edu

Key words: peritonitis, abdominal cocoon, sclerosing encapsulating peritonitis, Intestinal obstruction.

Contributions: JJP, concept and manuscript writing and editing; NT, data collection and manuscript writing; MBF, critical revision of the manuscript.

Conflicts of interest: the authors have no conflict of interest to report.

Received for publication: 20 October 2011. Accepted for publication: 22 February 2012.

This work is licensed under a Creative Commons Attribution NonCommercial 3.0 License (CC BYNC 3.0).

CCopyright J.J. Pan et al., 2012

Licensee PAGEPress, Italy

Gastroenterology Insights 2012; 4:e3

doi:10.4081/gi.2012.e3

of SBP. He was started on ceftriaxone treatment. An upper endoscopy was performed that revealed grade II esophageal varices and band ligation was performed. He was discharged with prophylactic propranolol, diuretics and ciprofloxacin.

He presented with 5 episodes of small amount (1-2 cups) of hematemesis without abdominal pain to the hospital later, including the admission before his death. One of the episodes happened during a stress echocardiography, which was a part of pre-LT evaluation. During the repeated endoscopies, small superficial esophageal ulcers from the previous banding were the only significant findings. After receiving transfusions, he stayed in the hospital for 3-4 days on average. In mid January 2011, he presented with another episode of small amount of hematemesis along with nausea and worsening severe colicky abdominal pain. On physical examination, he had abdominal tenderness with guarding. A CT scan of the abdomen and pelvis was performed that showed marked dilatation of the esophagus, stomach, and the entire small bowel with apparent fecalization of the distal small bowel loops. Appearance of cocooning of the distal small bowel loops in the lower left midabdomen was unchanged. There was apparent twisting of the mesentery within the pelvis along with the suggestion of twisting of the 
sigmoid colon, both unchanged from the prior studies. The cause and transition point of the obstruction was not identified and no pneumatosis was seen (Figure 2). A nasogastric tube was then placed and wall suction was applied to decompress the bowel. In the mean time, he was put on the waiting list for LT due to an increasing model for end-stage liver disease score. Despite being managed conservatively, his leukocytosis worsened and he developed lactic acidosis. An emergent exploratory laparotomy was performed. Gangrenous closed loop small bowel obstruction in the setting of SEP was found and small bowel resection with diversion was performed. He remained in critical conditions after the surgery and passed away secondary to multi-organ failure 5 days after the surgery.

\section{Discussion}

According to single-center studies, SEP is a relatively rare entity in patients receiving $\mathrm{PD}$ with the prevalence ranges from $0.5 \%$ to $4.4 \%$. Nevertheless, the duration of PD has been reported a risk for SEP. The prevalence increased progressively from $1.9,6.4,10.8$, to $19.4 \%$ for patients being on dialysis for $2,5,6$, and 8 years, respectively. ${ }^{9}$ On the other hand, SEP seems to be more common in cirrhotic patients receiving PVS. Stanley and colleagues ${ }^{2}$ reported that 26 (38\%) of 69 cirrhotic patients with PVS compared with only 1 of 485 patients without PVS had SEP during autopsy.

The etiology and pathogenesis of SEP are unknown. The condition is proposed to be related to the persistent expression of transforming growth factor beta (TGF-b) on mesothelial cells. ${ }^{9}$ In 24 idiopathic cases, Wei and colleagues ${ }^{10}$ reported that omentum dysplasia occurred in $41.7 \%$ of them. They hypothesized that during embryonic development, the membranous greater omentum descends along the transverse colon and encases the intestine, thus forming the fibrous membrane like a cocoon. In the setting of PD, the number of abdominal surgeries, related or not to the catheter, might be a risk factor. Peritoneal exposure to glucose, hypertonicity, low $\mathrm{pH}$, plasticizers, glucose degradation products by heat sterilization, and trauma from the catheter tip, have been implicated as risk factors for SEP. ${ }^{11}$ Above all the risk factors, the most convincing ones are the use of chlorhexidine as a spray disinfectant for PD connectors and severe/nonresolving peritonitis. ${ }^{1}$ Ohmori and colleagues ${ }^{12}$ proposed that either CD34positive and/or CD117-positive mesenteric stem cells can proliferate and differentiate into myofibroblasts or fibroblasts that produce matrix proteins that gradually leads to SEP during continual irritation of PD.

In the setting of PVS, Stanley and colleagues $^{2}$ proposed that the increased flow through the abdominal cavity of ascites containing increased concentrations of plasma proteins cause greater fibrin clot formation and deposition of fibrin and other ascitic fluid proteins upon the peritoneal surface. The accelerated ascitic flow may facilitate spread through this medium of fibrogenic cytokines from sites of localized injuries, infections, or inflammations, to the general peritoneal cavity. In addition to increased deposition of fibrin, it is possible that some patients with SEP may also have defects in fibrinolytic activities. Similar to PD, it is not uncommon for decompensated cirrhotic patients to have recurrent $\mathrm{SBP}$, which is also considered a risk for SEP. ${ }^{5}$ Nevertheless, SEP can occur in cirrhotic patients without a history of SBP. ${ }^{7}$ In a small series, 5 patients developed SEP at variable time after LT. All of them had a normal laparotomy at the time of LT. Low-grade intra-abdominal sepsis was proposed to be the cause. ${ }^{8}$ Beta blockers have been remotely reported as a rare culprit for SEP in single-case reports. ${ }^{13-15}$

The presenting symptoms and signs of SEP such as nausea, vomiting, fullness, absent bowel sounds, abdominal pain, abdominal or pelvic mass and other clinical aspects are often nonspecific that may be seen in a variety of conditions. ${ }^{1}$ SEP is therefore often diagnosed at laparotomy or autopsy. Stanley and colleagues $^{2}$ reported that 12 of 26 (46\%) of their patients with SEP had intestinal obstruction which was the only manifestation and was fatal in 5. In another study, Wei and colleagues ${ }^{10}$ reported that the main manifestations among their patients were partial or complete intestinal obstruction (87.5\%) and abdominal mass (54.2\%). Only 4 of 24 patients were diagnosed preoperatively by radiographic studies and 20 were diagnosed by laparotomy. SEP is associated with a mortality rate as high as $56 \% .^{16}$ It is imperative to diagnose SEP before it causes serious problems such as bowel perforation. ${ }^{6}$

Ultrasound has been reported as a rapid and sensitive means for diagnosis of SEP. ${ }^{17}$ The earliest and most common finding is increased peristalsis affecting several bowel loops. As the condition progresses, the bowel becomes increasingly tethered together posteriorly. The characteristic pre-visceral membrane which encases the tethered and matted bowel loops is demonstrated only in the late stages of the disease. ${ }^{17}$ On CT scans, peritoneal thickening, peritoneal calcification, loculated fluid collections, and tethering of the small bowel appear to be diagnostic of SEP. More importantly, in a

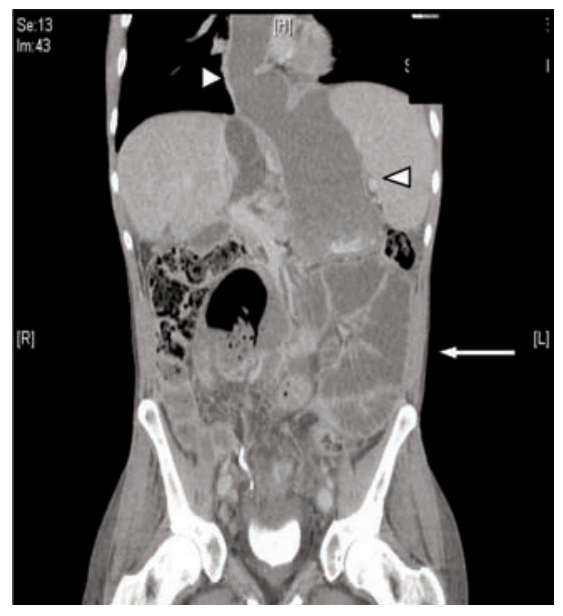

Figure 2. Coronal view of the computed tomography scan of patient \#2. Cluster of intestinal loops was seen in the left lower mid-abdomen (white arrow). In addition, the esophagus and stomach were extremely dilated (white arrow heads). 
small group of patients with follow-up CT scans, new or progression of, CT findings correlated with clinical severity of SEP. ${ }^{18}$ Nevertheless, Tarzi and colleagues ${ }^{19}$ suggested that CT scanning may not be useful as a screening tool for SEP as the scan is frequently normal even a few months before the fulminant illness. ${ }^{19}$ Another possible explanation for their findings is that CT scanning may not be sensitive enough to detect early SEP. In a small series, Kropp and colleagues ${ }^{20}$ diagnosed SEP in 3 PD patients with laparoscopy. All of them had signs and symptoms suggestive of SEP but had normal abdominal CT scans. In summary, SEP often has nonspecific signs and symptoms and has high mortality rates. It is possible although difficult to diagnose SEP preoperatively based on the clinical and radiological features, in the absence of other plausible etiologies for intestinal obstruction.

Surgical intervention with dissection and excision of the fibrous membrane from the intestine is often required for patients with SEP complicated with small bowel obstruction. However, the prognosis after surgery is usually poor. ${ }^{1}$ Multiple reports have shown that patients with SEP can be managed conservatively consisting of corticosteroids and/or immunosuppressive drugs with or without total parenteral nutrition (TPN). Junor and colleagues $^{21}$ first reported immunosuppressive therapy is associated with prolonged survival in patients with SEP after renal transplantation. In a small series, 11 patients on PD were diagnosed with SEP. Five of them receiving prednisolone remained alive while the others not on the medication died. The authors suggested that steroid therapy should be considered as a first line therapy for PD patients with SEP. ${ }^{22}$ Preoperative administration of immunosuppression to patients with SEP has been suggested. ${ }^{23}$ In a series of PD patients, 23 patients were diagnosed with peritoneal sclerosis. Among them, 9 received tamoxifen and 14 received no treatment (control). No patient treated with tamoxifen developed SEP while 4 in the control group developed SEP and died. The mechanism of tamoxifen in the prevention of SEP is unknown. ${ }^{24}$ In a rat model for SEP,25 thalidomide reduced the extent and severity of histological signs of peritoneal injury, which could be a potential treatment for patients with SEP.

Corticosteroids can benefit cirrhotic patients with SEP. Yamamoto and colleagues ${ }^{5}$ reported that a single cirrhotic patient with SEP and intestinal obstruction was successfully treated with prednisolone $(5 \mathrm{mg} /$ day) after total enterolysis. The patient continued to be symptom free at 15 months after the surgery. They proposed immunosuppressive therapy may prevent recurrence of SEP. In another case report, ${ }^{7}$ a patient was diagnosed SEP during laparotomy for LT. The surgery was deferred and the patient was started on prednisone, azathioprine and tamoxifen, followed by a successful LT 7 days later despite the presence of the same thickened peritoneum. Based on the previous findings, immunosuppressive therapy seems to be able to alleviate and/or prevent the recurrence of SEP. Nevertheless, Lin and colleagues $^{3}$ reported a single case developing SEP 2 weeks after LT despite large doses of immunosuppressants (tacrolimus, mycophenolate mofetil, and methylprednisolone). This patient had PVS and SEP prior to the LT. The authors recommended that SEP should be cleared away during LT to prevent any following possible intestinal obstruction. On the other hand, immunosuppression may need to be adjusted if SEP is diagnosed during LT since calcineurin inhibitor such as cyclosporine and tacrolimus may accelerate SEP. ${ }^{7}$ In an animal model, tacrolimus has been shown to increase TGF-b1 expression. ${ }^{26}$ This finding can be translated to increased fibrogenesis, which theoretically can accelerate SEP.

In conclusion, SEP is a rare cause of intestinal obstruction and carries a high mortality rate. The pathogenesis is still unknown although many theories exist. Its manifestations are often vague and nonspecific that may lead to misdiagnosis or delayed diagnosis. It is possible, although difficult, to diagnose SEP preoperatively based on the clinical and radiological features, in the absence of other plausible etiologies for intestinal obstruction. The condition is often diagnosed at laparotomy or autopsy. For patients with SEP and bowel obstruction, surgical treatment is often required. Nevertheless, patients with this problem can be treated conservatively with immunosuppressive therapy with or without TPN before going for surgery.

\section{References}

1. Kawaguchi Y, Kawanishi H, Mujais S, et al. Encapsulating peritoneal sclerosis: definition, etiology, diagnosis, and treatment. International Society for Peritoneal Dialysis Ad Hoc Committee on Ultrafiltration Management in Peritoneal Dialysis. Perit Dial Int 2000;20Suppl4:S43-55.

2. Stanley MM, Reyes CV, Greenlee HB, et al. Peritoneal fibrosis in cirrhotics treated with peritoneovenous shunting for ascites. An autopsy study with clinical correlations. Dig Dis Sci 1996;41:571-7.

3. Lin CH, Yu JC, Chen TW, et al. Sclerosing encapsulating peritonitis in a liver transplant patient: a case report. World J Gastroenterol 2005;11:5412-3.

4. Yamada S, Tanimoto A, Matsuki Y, et al. Sclerosing encapsulating peritonitis (abdominal cocoon) associated with liver cirrhosis and diffuse large B-cell lymphoma: autopsy case. Pathol Int 2009;59: 681-6.

5. Yamamoto S, Sato Y, Takeishi T, et al. Sclerosing encapsulating peritonitis in two patients with liver cirrhosis. J Gastroenterol 2004;39:172-5.

6. Wakabayashi H, Okano K, Suzuki Y. Clinical challenges and images in GI. Image 2. Perforative peritonitis on sclerosing encapsulating peritonitis (abdominal cocoon) in a patient with alcoholic liver cirrhosis. Gastroenterology 2007;132:854.

7. de Vos Tot Nederveen Cappel WH, Dubbeld J, Willems SM, et al. Liver transplantation in a patient with encapsulating peritoneal sclerosis. Neth J Med 2008;66:280-2.

8. Maguire D, Srinivasan P, O'Grady J, et al. Sclerosing encapsulating peritonitis after orthotopic liver transplantation. Am J Surg 2001;182:151-4.

9. Dobbie JW. Pathogenesis of peritoneal fibrosing syndromes (sclerosing peritonitis) in peritoneal dialysis. Perit Dial Int 1992;12:14-27.

10. Wei B, Wei HB, Guo WP, et al. Diagnosis and treatment of abdominal cocoon: a report of 24 cases. Am J Surg 2009;198: 348-53.

11. Hendriks PM, Ho-dac-Pannekeet MM, van Gulik TM, et al. Peritoneal sclerosis in chronic peritoneal dialysis patients: analysis of clinical presentation, risk factors, and peritoneal transport kinetics. Perit Dial Int 1997;17:136-43.

12. Ohmori T, Ohnishi S, Okada K, Arita N. Sclerosing encapsulating peritonitis and non-occlusive mesenteric infarction found at autopsy in a man who had undergone continuous ambulatory peritoneal dialysis: a histochemical and immunohistochemical study. Pathol Int 2000;50:660-6.

13. Brown P, Baddeley H, Read AE, et al. Sclerosing peritonitis, an unusual reaction to a beta-adrenergic-blocking drug (practolol). Lancet 1974;2:1477-81.

14. Clark CV, Terris R. Sclerosing peritonitis associated with metoprolol. Lancet 1983;1: 937.

15. Ahmad S. Sclerosing peritonitis and propranolol. Chest 1981;79:361-2.

16. Rigby RJ, Hawley CM. Sclerosing peritonitis: the experience in Australia. Nephrol Dial Transplant 1998;13:154-9.

17. Hollman AS, McMillan MA, Briggs JD, et al. Ultrasound changes in sclerosing peritonitis following continuous ambulatory peritoneal dialysis. Clin Radiol 1991;43:176-9.

18. Stafford-Johnson DB, Wilson TE, Francis IR, Swartz R. CT appearance of sclerosing peritonitis in patients on chronic ambulatory peritoneal dialysis. J Comput Assist Tomogr 1998;22:295-9. 
19. Tarzi RM, Lim A, Moser S, et al. Assessing the validity of an abdominal CT scoring system in the diagnosis of encapsulating peritoneal sclerosis. Clin J Am Soc Nephrol 2008;3:1702-10.

20. Kropp J, Sinsakul M, Butsch J, Rodby R. Laparoscopy in the early diagnosis and management of sclerosing encapsulating peritonitis. Semin Dial 2009;22:304-7.

21. Junor BJ, McMillan MA. Immun-osuppression in sclerosing peritonitis. Adv Perit
Dial 1993;9:187-9.

22. Kuriyama S, Tomonari H. Corticosteroid therapy in encapsulating peritoneal sclerosis. Nephrol Dial Transplant 2001;16: 1304-5.

23. Bhandari S, Wilkinson A, Sellars L. Sclerosing peritonitis: value of immunosuppression prior to surgery. Nephrol Dial Transplant 1994;9:436-7.

24. del Peso G, Bajo MA, Gil F, et al. Clinical experience with tamoxifen in peritoneal fibrosing syndromes. Adv Perit Dial 2003; 19:32-5.

25. Mondello S, Mazzon E, Di Paola R, et al. Thalidomide suppresses sclerosing encapsulating peritonitis in a rat experimental model. Shock 2009;32:332-9.

26. Maluccio M, Sharma V, Lagman M, et al. Tacrolimus enhances transforming growth factor-betal expression and promotes tumor progression. Transplantation 2003; 76:597-602. 